TITLE:

Characteristic Energy Loss Spectra and Optical Constants of Some Solid Hydrocarbons(Dissertation_全文)

$\operatorname{AUTHOR}(S)$ :

Okabe, Toshio

CITATION:

Okabe, Toshio. Characteristic Energy Loss Spectra and Optical Constants of Some Solid Hydrocarbons. 京都大学, 1974, 理学博士

ISSUE DATE:

1974-01-23

URL:

https://doi.org/10.14989/doctor.k1429

RIGHT: 


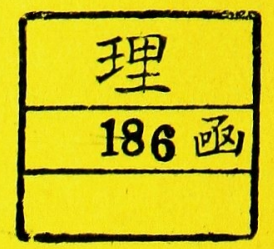

学位申請論文

周部俊夫 


\section{本論丈}

Characteristic Energy Loss Spectra and Optical Constants of Some Solid Hydrocarbons

Toshio Okabe: J. Phys. Soc. Japan 35, in press.

\section{参考論丈 1}

Diffuse Scattering of Electrons by Defect in Crystal.

I: General Formulation and Application to Guinier-Preston Zone

Toshio Okabe, Katsuhiko Hasebe and Michi-hiko Mannami:

J. Phys. Soc. Japan 27 (1969) 1245.

\section{参考論文 2}

Diffuse Scattering of Electrons by Defect in Crystal. II. Structure Analysis of the Guinier-Preston Zone in $\mathrm{Cu}-\mathrm{Be}$ alloy

Katsuhiko Hasebe, Toshio Okabe and Michi-hiko Mannami:

J. Phys. Soc. Japan 30 (1971) 417 . 


\title{
Characteristic Energy Loss Spectra and Optical Constants of Some Solid Hydrocarbons
}

\section{Toshio OKABE}

\author{
Department of Physics, Faculty of Science, \\ Kyoto University
}

Characteristic electron energy loss measurements on some solid hydrocarbons, $\beta$-carotene, diphenyl-polyenes and paraffins applying $40 \mathrm{keV}$ electrons are reported. From the energy loss function $-\operatorname{Im}[1 / \varepsilon(\Delta E)]$ the dielectric constant $\varepsilon(\Delta E)$ is calculated by means of a Kramers-Kronig transformation. It is possible to divide the optical properties of these compounds into three spectral regions. The low-lying losses below $8 \mathrm{eV}$ observed in $\beta$-carotene and diphenyl-polyenes are interpreted by the single excitation of $\pi$-electrons. In the range 8 to $16 \mathrm{eV}$ the excitations of $\sigma$-electrons are observed. The electron loss at $21 \mathrm{eV}$ is due to the plasma oscillations of $\pi$ - and $\sigma$-electrons. 
$\S 1$. Introduction

The electronic structures of organic compounds have been studied by the optical absorption methods in the VUV region up to about $7 \mathrm{eV}^{1}$ and recently their excited electronic states in higher energy region have been made clear with the optical reflectivity measurements and also with the characteristic electron energy loss experiments.

The electron energy loss experiments have been widely adopted to investigate the aromatic hydrocarbons and some polymers. Jäger $^{2)}$ interpreted the loss peak at about $6 \mathrm{eV}$ in the energy loss spectra of the aromatic hydrocarbons as a hybrid resonance effected both by single $\pi$-electron excitations and collective interactions, while otto and coworkers ${ }^{3 \sim 5)}$ identified the loss as a $\pi-\pi^{*}$ single-electron transition in comparison with optical absorption measurements. In polystyrene the existence of the collective effect at about $7 \mathrm{eV}$ was confirmed in terms of the energy dependent dielectric constant derived from the energy loss spectra, ${ }^{6)}$ which was supported later in the optical reflectivity measurements. 7 )

In the case of long chain compounds, the investigations have been carried optically only in the low energy region. Much attentions have been paid on the convergence of the wavelengh of optical absorption maxima observed in this region for polyenes and asymmetric cyanines as the number of carbon atoms increased and this has been interpreted in terms of the single excitation of $\pi$-electrons. 8 )

This paper reports the characteristic electron energy loss 
spectra of some long chain hydrocarbons having carbon conjugated double bonds; $\beta$-carotene, diphenyl-polyenes and those of normal paraffins. Then the loss spectra are transformed into the optical constants by applying the Kramers-Kronig dispersion relation. From both the electron loss spectra and the optical constants the excitations of $\pi$ - and $\sigma$-electrons are discussed.

\section{§2. Experimental}

Specimen films were prepared from $\beta$-carotene $\mathrm{C}_{40} \mathrm{H}_{56}$, diphenyl= polyenes $\mathrm{C}_{6} \mathrm{H}_{5}(-\mathrm{CH}=\mathrm{CH}-){ }_{n} \mathrm{C}_{6} \mathrm{H}_{5}$ for $\mathrm{n}=2,3,4$ and 26-, 36-normal= paraffins. Diphenyl-polyenes and paraffins were vacuum evaporated onto mica, then the thin films were floated on the water and scooped with copper mesh. Another technique was used for $\beta$-carotene; the benzene solution of about one per cent by weight was dropped on the water by glass squirt to form thin film and the film was scooped with the mesh for specimen holder. This procedure was done in argon atmosphere in the dark box to prevent the oxidization of $\beta$-carotene. To examine the change of specimens during the preparation of thin films, the optical absorptions of thin films dissolved in organic solutions were measured. It was found that the specimen chemical structres were unchanged during the preparations of thin films.

The energy loss spectra were obtained by the Möllenstedt type energy analyzer attached to an electron microscope. The incident electron energy was $40 \mathrm{keV}$ and the energy resolution of the analyzer was about $1 \mathrm{eV}$. The energy analysis was made by forming the electron microscope image with the objective aperture which accepted 
the electrons scattered in the angle less than $3 \mathrm{mrad}$. The energy spectra were recorded on the photographical plates and the intensity was measured by the microphotometry. Energy calibration was made by employing the plasma loss of aluminum for $15.0 \mathrm{eV}$, and the linearity check of the energy scale was made folowing the procedure by Swanson and Powell. 9 )

Also the optical absorption spectra of the solid films of the materials evaporated onto mica or quartz were measured in the low energy region below $6 \mathrm{eV}$ in order to compare with the optical constants derived from the energy loss spectra.

\section{§3. Results}

Some of the observed electron energy loss spectra are shown in Fig. I and the loss values are tabulated in Table I. Also the errors of the loss values are estimated in Table I. The accuracy of the profiles of the loss spectra are observed better than $10 \%$ for each energy values. The electron loss spectra of the compounds studied show a similar profile in the energy region higher than about $8 \mathrm{eV}$; a very broad peak at about $21 \mathrm{eV}$ and a shoulder or a peak at about $10 \mathrm{eV}$. Carotene and diphenyl-polyenes, which have carbon conjugated double bonds, show peaks at lower energy region; a peak at $3 \mathrm{eV}$ for $\beta$-carotene and two peaks at $4 \mathrm{eV}$ and $7 \mathrm{eV}$ for diphenyl-polyenes. However, paraffins which have only carbon linear single chains indicate no remarkable peak in this energy region.

The observed loss spectra can be correlated with the optical properties. The differential cross section of the incoming electrons for the transfer of momentum $t k$ (scattering angle $\theta$ ) and 
energy $\hbar \omega=\Delta \mathrm{E}$ into the solid angle $\mathrm{d} \Omega$ is proportional to the imaginary part of the inverse dielectric constant (the loss function) as 10 )

$$
\frac{d^{2} \sigma}{d(\hbar \omega) d \Omega}=\frac{1}{2 \pi^{2} a_{0} n E_{0}} \frac{1}{\theta^{2}+\left(\hbar \omega / 2 E_{0}\right)^{2}} \operatorname{Im} \frac{-1}{\varepsilon(k, \omega)}
$$

where $E_{0}$ is the energy of the incident electrons, $a_{0}$ is Bohr radius and $\mathrm{n}$ is the number of electrons per volume participating in the process. In the present experimental conditions the observed loss spectra were correlated to be proportional to the cross section integrated over the scattering angle as

$$
\begin{aligned}
\frac{d \sigma}{d(\hbar \omega)} & =\frac{1}{2 \pi^{2} a_{0} n E_{0}} \int_{0}^{\theta_{L}} \frac{1}{\theta^{2}+\left(\hbar \omega / 2 E_{0}\right)^{2}} d \Omega \operatorname{Im} \frac{-1}{\varepsilon(\omega)} \\
& =\frac{1}{2 \pi a_{0} n E_{0}} \log \left\{1+\left(\frac{2 E_{0} \theta_{L}}{\hbar \omega}\right)^{2}\right\} \operatorname{Im} \frac{-1}{\varepsilon(\omega)}
\end{aligned}
$$

where $\theta_{L}$ (the maximum angle of the scattered electrons captured by the objective aperture into the loss spectrum) is 3 mrad. Moreover, for the loss function there exists the f-sum rule;

$$
\int_{0}^{\infty} \omega \operatorname{Im} \frac{-1}{\varepsilon(\omega)} d \omega=\frac{2 \pi^{2} e^{2} N}{m} n_{0}
$$

where $\mathrm{N}$ is the density of atoms (or molecules) in the material, $\mathrm{n}_{0}$ is the number of electrons per atom (molecule); $\mathrm{Nn}_{0}=\mathrm{n}$, and $\mathrm{m}$ is the free electron mass. Then the observed loss spectra give the loss function $-\operatorname{Im}[1 / \varepsilon(\omega)]$ according to eqs. (2) and (3). The real part of $1 / \varepsilon(\omega)$ are derived by employing the Kramers-Kronig dispersion integration as ${ }^{11)}$

$$
\operatorname{Re} \frac{1}{\varepsilon(\omega)}=1+\frac{2}{\pi} \mathrm{P} \int_{0}^{\infty} \frac{\omega^{\prime} \operatorname{Im}\left[1 / \varepsilon\left(\omega^{\prime}\right)\right]}{\omega^{\prime 2}-\omega^{2}} d \omega^{\prime}
$$


where $P$ indicates the principal-part integral. The integration was computed numerically over the energy range ou $45 \mathrm{eV}$ dividing $0.1 \mathrm{eV}$ width. For the integration the energy loss spectra were corrected as shown by dashed lines in Fig. I to avoid the effects on no-loss electrons and those of multiple scattering above $30 \mathrm{eV}$. Also in the present work to normalize the loss function the value $n_{0}$ was chosen so that the one of the resultant dielectric constants $\varepsilon_{2}$ agree qualitatively with the optical absorption measurements in the range below $6 \mathrm{eV}$ (Fig. 3). It was found that the agreement between the calculated values and the optical measurements is good in the case when only carbon atoms should participate in the interaction. Some of these optical constants $\varepsilon_{1}, \varepsilon_{2}$ and $-\operatorname{Im}(1 / \varepsilon)$ are shown in Fig. 2. The behaviors of the imaginary parts of the dielectric constants correspond well with their energy loss spectra or their loss functions in the region below $8 \mathrm{eV}$ and the peaks of $\varepsilon_{2}$ at approximately $10 \mathrm{eV}$ correspond to the shoulders in the electron loss spectra. The imaginary parts of the dielectric constants show peaks in the low energy region for $\beta$-carotene and diphenyl-polyenes. On the other hand there is no peak for paraffins in this region.

\section{§4. Discussion}

The low-lying electron energy loss peaks of $\beta$-carotene at 3.0 $\mathrm{eV}$ and diphenyl-polyenes at $4 \mathrm{eV}$ can be corresponded to the optical absorption peaks arising from the single excitation of $\pi$-electrons. 8 , The dielectric constants $\varepsilon_{2}$ derived here have peaks at $2.7 \mathrm{eV}$ for $\beta-$ carotene and $3.6 \mathrm{eV}$ for diphenyl-hexatriene, whose values are in good agreement with the optical absorption measurements of these solid films as $2.77 \mathrm{eV}$ and $3.83 \mathrm{eV}$ respectively (Fig. 3). The 
interpretation of thse losses from the standpoint of the collective excitations of $\pi$-electrons ${ }^{13}$ ) cannot be accepted since the dielectric constants $\varepsilon_{1}$ and $\varepsilon_{2}$ are too large to satisfy the condition for the excitation of plasma oscillations; $\varepsilon \cong 0$. It seems that the only example in which the plasma oscillation of $\pi$-electrons has been established is the $7 \mathrm{eV}$ loss of graphite. 14,15)

Two peaks observed between 8 to $16 \mathrm{eV}$ on the curves of $\varepsilon_{2}$ in Fig. 2 may be ascribed to the excitations o-electrons; (CH)-(CH)* excitations and $\sigma-\sigma^{*}$ excitations involving the $\mathrm{C}-\mathrm{C}$ bonds. The lower peaks in this energy region give approximately the same energy values through the series. The location of the higher peaks, however, moves upwards in energy as the distance of $\mathrm{C}-\mathrm{C}$ bonds decreases. Therefore we expect the $\sigma-\sigma^{*}$ excitations to lie at higher energy than $(\mathrm{CH})-(\mathrm{CH}) *$ excitations. Honig ${ }^{16}$ ) showed that the ionization potentials (the highest occupied levels) of o-electrons were observed from propane to decane as $11.21 \mathrm{eV}$ to $10.19 \mathrm{eV}$, which are consistent with the present experiment.

In the region above $16 \mathrm{eV}$ the dielectric constants $\varepsilon_{1}$ and $\varepsilon_{2}$ are monotonously increasing and decreasing respectively similar to the Drude's formula for the free electron gas. Therefore we may expect the free electron like behavior of the systems in this region. So the broad peaks at $21 \mathrm{eV}$ in the loss functions can be interpreted as those due to the excitations of plasmon as in the case of metals. In fact the behaviors of the dielectric constants in this region confirm this mode of excitations where $\varepsilon_{1}$ and $\varepsilon_{2}$ are small. In graphite the loss at $25 \mathrm{eV}$ are assigned as plasma loss of the $\pi+\sigma$-electrons ( 4 per atoms). 14) However, the loss values in the present work do not coincide well with the values calculated from 
the free-electron model according to $\hbar \omega_{p}=\hbar \sqrt{4 \pi N n_{0} e^{2} / m}$. These values are shown in Table II. The observed loss values are in the second column. The values in the third and the forth columns are calculated ones which count all the valence electrons and do only the valence electrons of carbons as $n_{0}$ respectively. The observed energies of plasma oscillations are higher than the calculated ones. It is known that the displacement of the plasma frequency to higher values is caused by a low-lying interband transitions. 17) Therefore the shift is ascribed to the interband transitions of o-electrons in the range 8 to $16 \mathrm{eV}$ and also this has been observed in the aromatic hydrocarbons. 2)

The losses at $7 \mathrm{eV}$ in the electron energy loss spectra which correspond to $6.1 \mathrm{eV}$ peaks in $\varepsilon_{2}$ are observed only in diphenyl= polyenes. The UV absorption spectra of benzene and its derivatives have been extensively measured and an intense absorption peak observed in the vicinity of $6 \mathrm{eV}$ has been ascribed to $\pi-\pi^{*}$ single electron excitation of the benzene ring m-electrons. ${ }^{18,19)}$ Therefore these losses can be interpreted as the excitation of $\pi$-electrons on the benzene rings at both ends of the carbon chains.

In the present electron loss experiments we only utilized the relative cross sections. Therefore to get the loss function $-\operatorname{Im}(I / \varepsilon)$ it was necessary to normalize the observed energy spectrum according to eq. (3). The profile of the dielectric constants obtained by employing the Kramers-Kronig relation is very sensitive to the choice of the value $n_{0}$. Only in the case that we used the number of electrons of carbon atoms without hydrogen atoms the derived $\varepsilon_{2}$ were in good agreement with the one from the optical absorption measurements. The same situation has been recognized 
for polystyrene in the works of Lavilla and Mendlowitz. ${ }^{6}$ The reason why only carbon atoms should participate in the excitations, however, has not been yet understood. Another method for the normalization of the loss function employed by Creuzburg, ${ }^{20}$ ) where the measurements of the thickness of the specimen films are used for this purpose, will be useful for the comparison with the present work. Furthermore it is necessary to compare the calculated dielectri constants from the electron energy loss spectrum with the optical measurements over the wide energy region.

Acknowledgements

The authir wishes to express his sincere thanks to Prof. Mannami for his encouragement and useful discussions during the course of this study. Thanks are also due to Dr. Yamashita for the Guidance to write the computer program for the $\mathrm{K}-\mathrm{K}$ dispersion integration and to Dr. Taniguchi for the optical measurements. 
References

1) UV-Atlas Organisher Verbindungen (Butherworth, Verlap, Chemie, $1966)$.

2) J. Jäger: Ann. Phys. (Germany) 22 (1969) 147.

3) S. Kunstreich and A. Otto: Optics Commun. 1 (1969) 45.

4) E. E. Koch and A. Otto: Opties Commun. 1 (1969) 47.

5) E. E. Koch, S. Kunstreich and A. Otto: Optics Commun. 2 (1971) 365 .

6) R. E. Lavilla and H. Mendlowitz: J. Phys. Radium 25 (1964) 114.

7) J. G. Carter, T. M. Jelinek, R. N. Hamm and R. D. Birkhoff:

J. chem. Phys. 44 (1966) 2266.

8) O. W. Adams and R. L. Miller: Theoret. chim. Acta 12 (1968) 151.

9) N. Swanson and C. J. Powell: J. chem. Phys. 39 (1963) 630.

10) P. Nozières and D. Pines: Phys. Rev- 113 (1959) 1254.

11) D. Pines: Elementary Excitations in Solids (Benjamin, New York, 1964) Chap. 3, p. 134.

12) H. H. Jaffe and M. Orchin: Theory and Applicatio of Ultraviolet Spectroscopy (John Wiley, New York, 1962) Chap. ll, p. 220

13) G. Araki and T. Murai: Prog. theor. Phys. 8 (1952) 639;

T. Murai: Prog. theor. Phys. 27 (1962) 899.

14) E. A. Taft and H. R. l'hiljpp: Phys.Rev-138 (1965) Al97.

15) K. Zeppenfeld: Z. Phys. 211 (1968) 391.

16) R. Je. Honis: J chem. Phys. 16 (1948) 1.05.

17) H. Raether: Springer Tracts in Modern Physics ed. G. Hohler (Springer, Berlin, 1:55) Vol. 38. p. 94.

18) V. J. Hammoni et al.: Jiscussicis Faraday Soc.9 (1950) 53.

- 19) S. Onari: j. Hnys soc J'pan 26 (jirig) 500.

20) M. Creuzburg: Z. Phis: 196 (196; : : 
Figure Captions

Fig. 1. Characteristic energy loss spectra. a) B-carotene;

b) diphenyl-hexatriene; c) 26-n-paraffin. The dotted curves indicate the profiles of the major energy loss.

Fig. 2. Dielectric constants $\varepsilon_{1}, \varepsilon_{2}$ and the energy loss functions $-\operatorname{Im}(I / \varepsilon)$ of a) $\beta-c$ arotene, b) diphenyl-hexatriene and c) 26-n-paraffin obtained by Kramers-Kronig analysis of the corrected curves (dashed lines) in Fig. 1.

FIg. 3. Optical absorption spectra of solid hydrocarbons. a) B-carotene, dried on LIF from the chloroform solution (solid line); b) diphenyl-hexatriene, vacuum evaporated on quartz (solid line). The dashed lines are the spectra of the chloroform solutions of the materials. 
Table I. Characteristic energy loss values (in eV).

\begin{tabular}{l|rrrr}
\hline substance & \multicolumn{4}{|c}{ loss values (in eV) } \\
\hline B-carotene & $3.0 \pm 0.2$ & & $10.5 \pm 0.2$ & $20.6 \pm 0.4$ \\
Diphenyl-butadiene & $4.1 \pm 0.1$ & $7.0 \pm 0.1$ & $11.1 \pm 0.2$ & $21.0 \pm 0.5$ \\
Diphenyl-hexatriene & $3.9 \pm 0.1$ & $7.0 \pm 0.1$ & $11.0 \pm 0.2$ & $21.4 \pm 0.2$ \\
Diphenyl-octatetraene & $3.7 \pm 0$ & $6.5 \pm 0.1$ & $10.4 \pm 0.1$ & $20.5 \pm 0.2$ \\
26-n-paraffin & & & $9.8 \pm 0.2$ & $20.2 \pm 0.4$ \\
$36-n$-paraffin & & & $96 \pm 0.2$ & $20.4 \pm 0.5$
\end{tabular}

Table II. Free electron plasmon energy $\hbar \omega_{p}$ cal compared with the observed $\hbar \omega_{p}$ obs (in eV).

\begin{tabular}{l|c|c|c}
\hline \multicolumn{1}{c|}{ substance } & $\hbar \omega_{\mathrm{p} \text { obs }}$ & $\hbar \omega_{\mathrm{p}} \mathrm{cal}^{*}$ & $\hbar \omega_{\mathrm{p} \mathrm{cal}}{ }^{*}$ \\
\hline B-carotene & $20.6 \pm 0.4$ & 18.2 & 15.7 \\
Diphenyl-butadiene & $21.0 \pm 0.5$ & 17.4 & 15.8 \\
Diphenyl-hexatriene & $21.4 \pm 0.2$ & 17.6 & 16.0 \\
Diphenyl-octatetraene & $20.5 \pm 0.2$ & 177 & 16.0 \\
$26-$-paraffin & $20.2 \pm 0.4$ & 18.8 & $15 . ?$ \\
$36-$ n-paraffin & $20.4 \pm 0.5$ & 18.8 & 15.3
\end{tabular}

* All valence electrons are taken into account to participate the excitation of plasmon.

* Only the valence electrons of carbon atoms are taken into account ' on this excitation. 
FIG. 1 a)

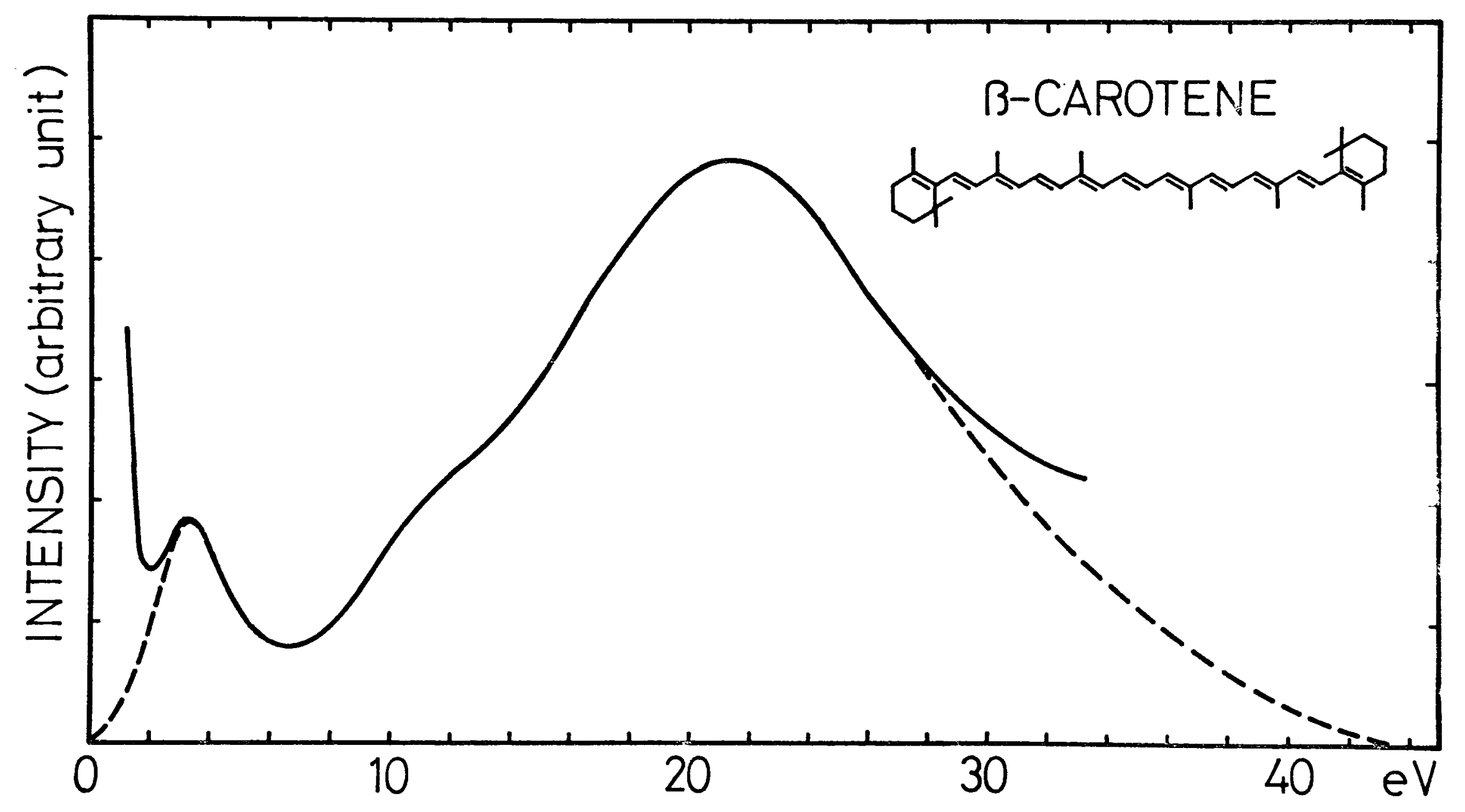


FIG. 1 b)

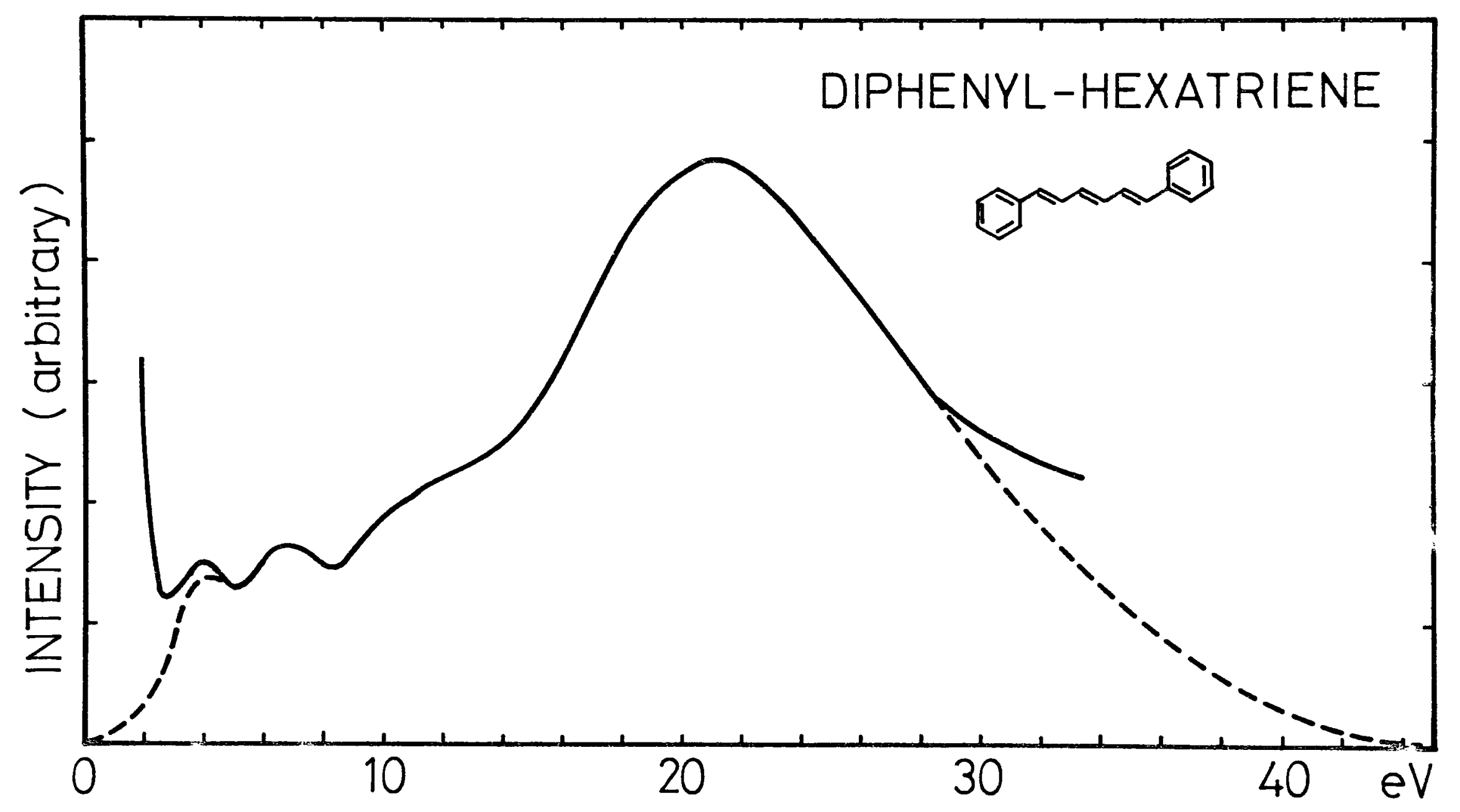


FIG. 1 c)

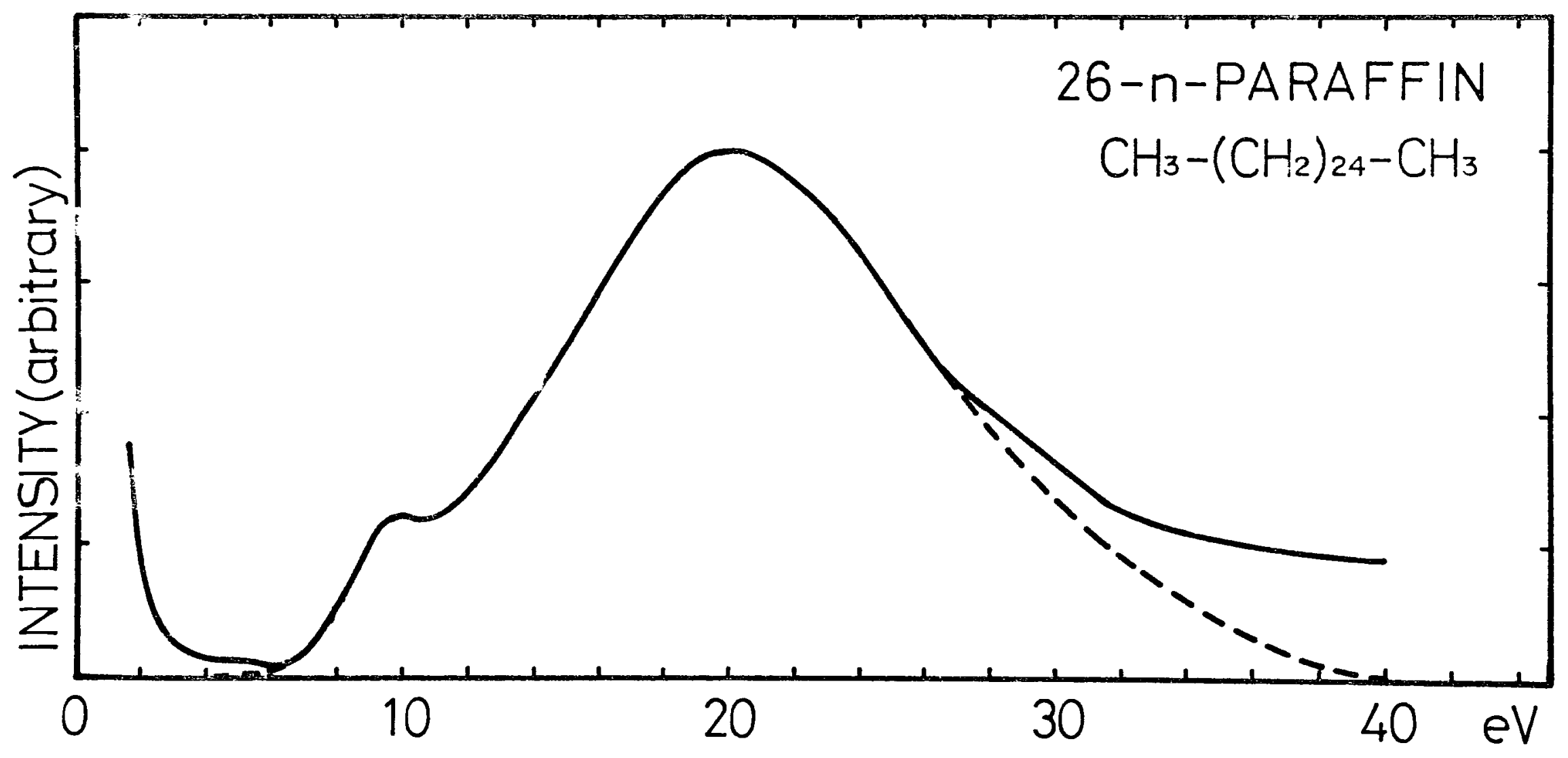


FIG. 2 a)

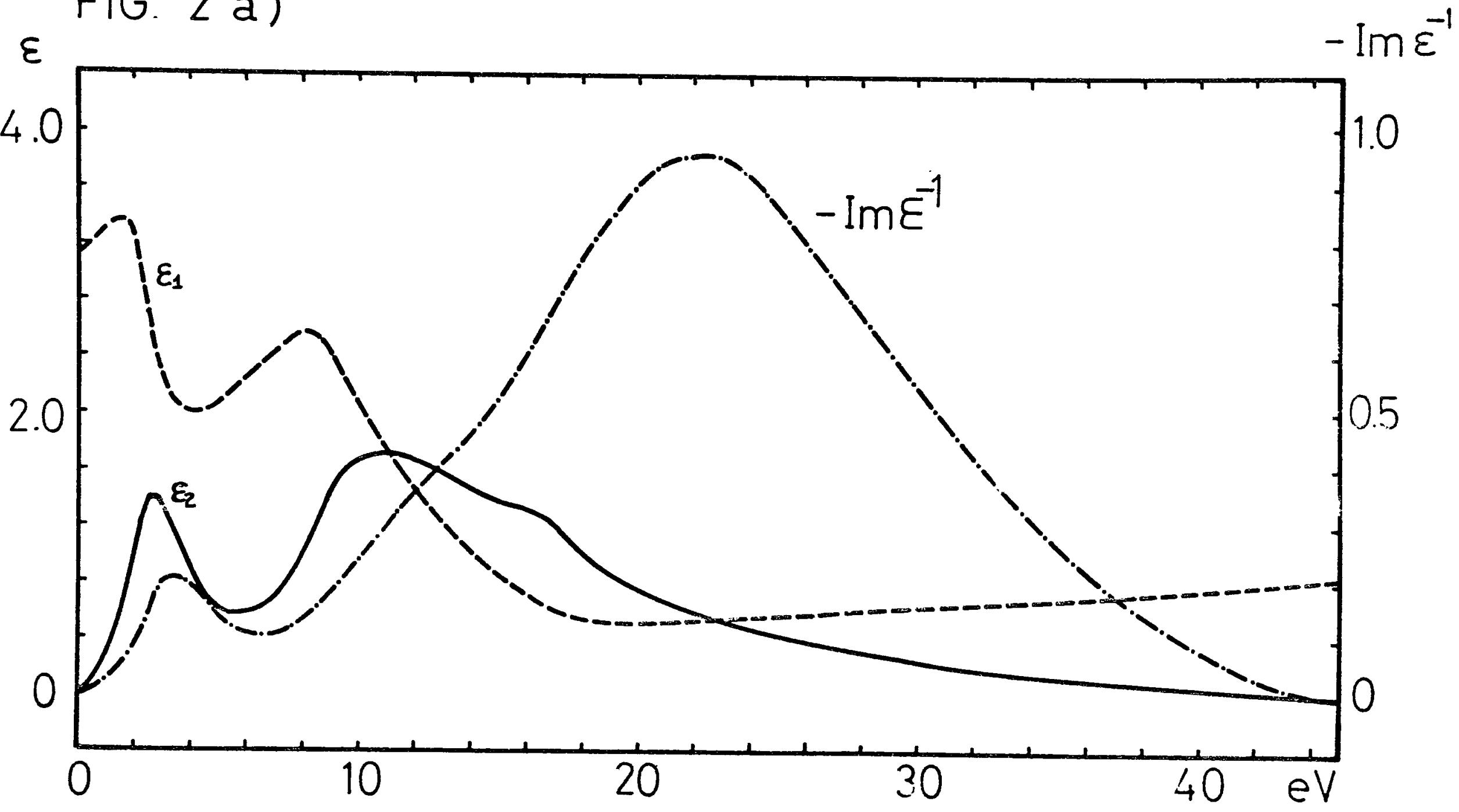


FIG. 2 b)

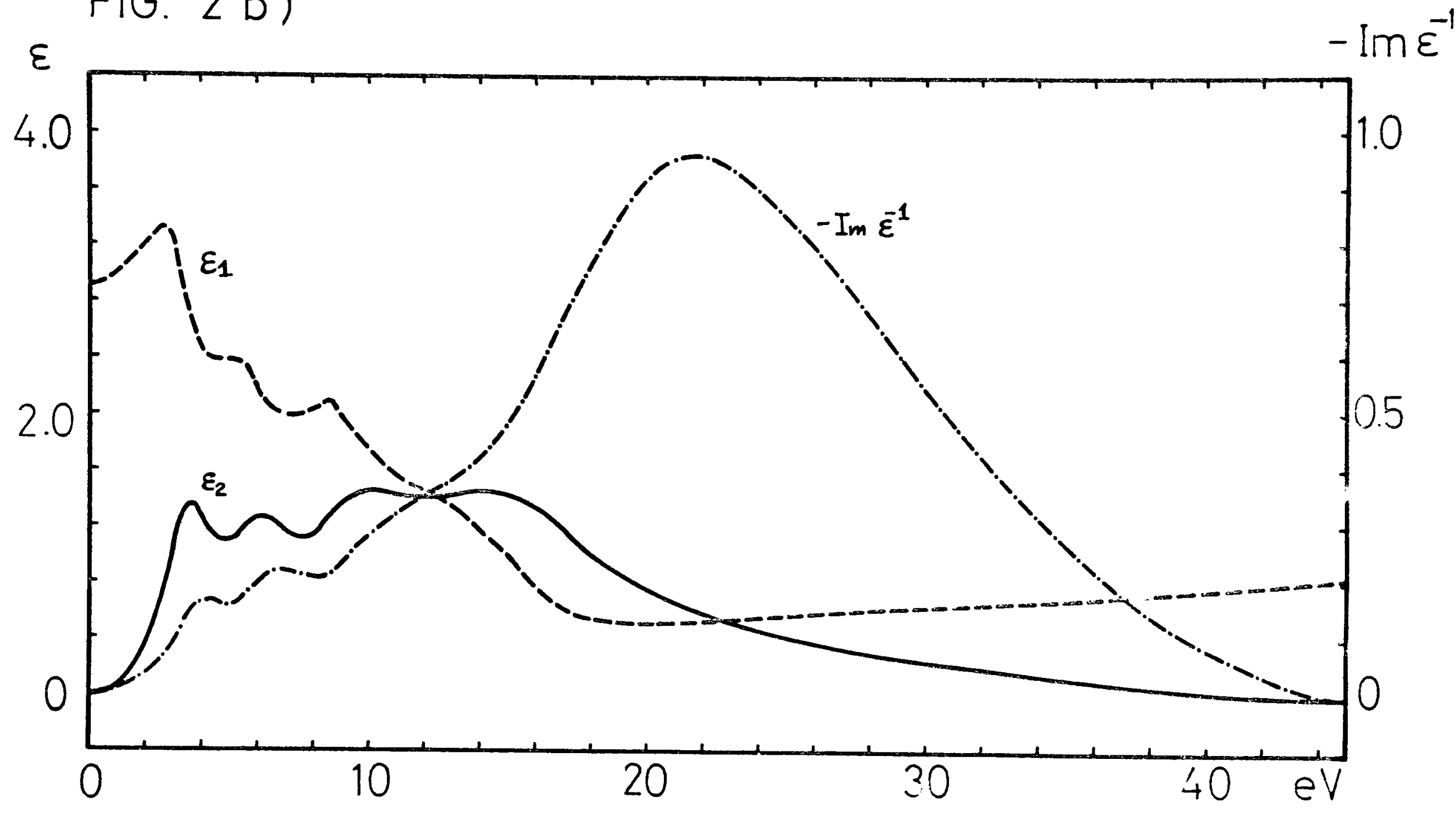


FIG. 2 c)

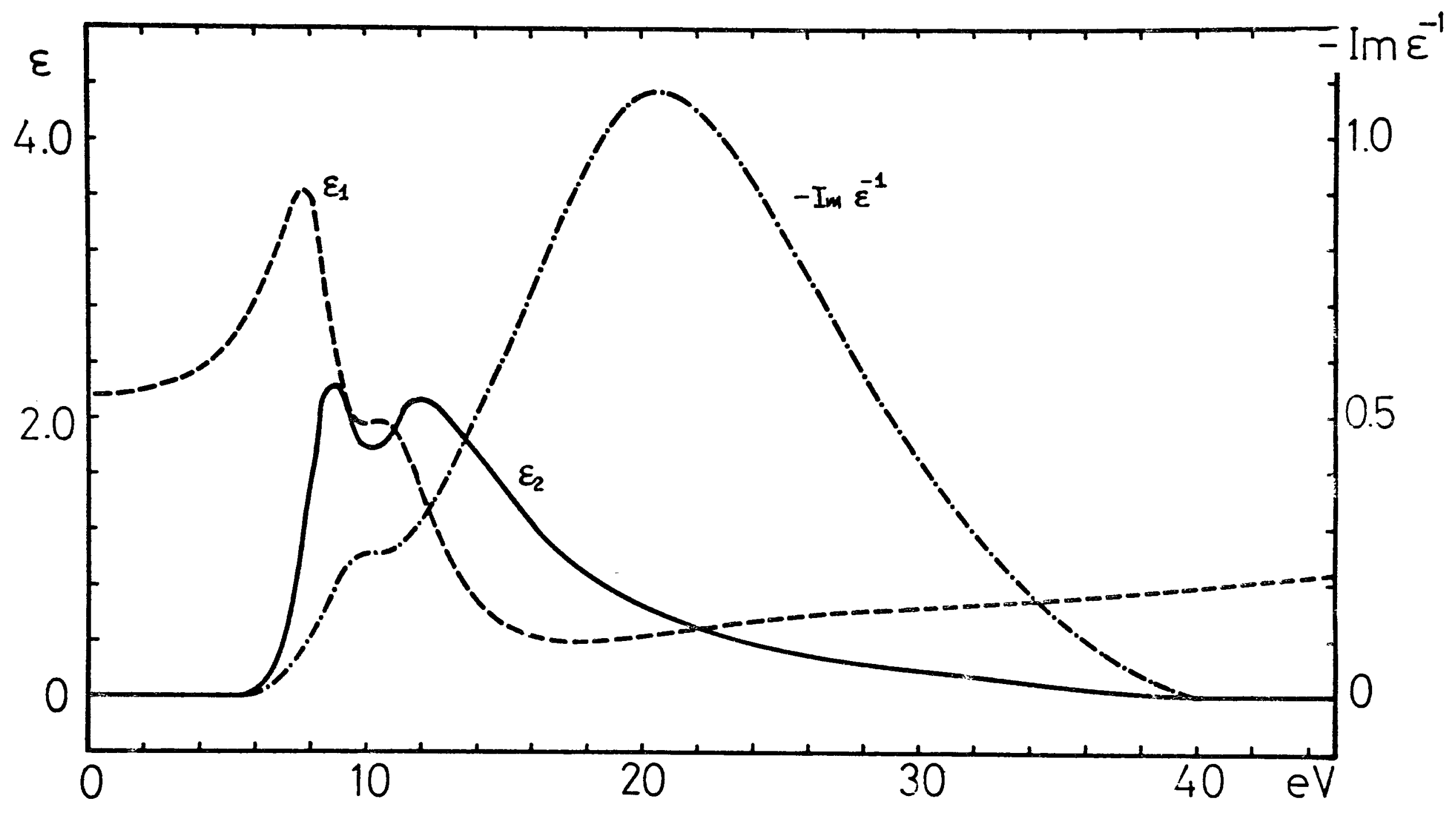


FIG. 3 a)

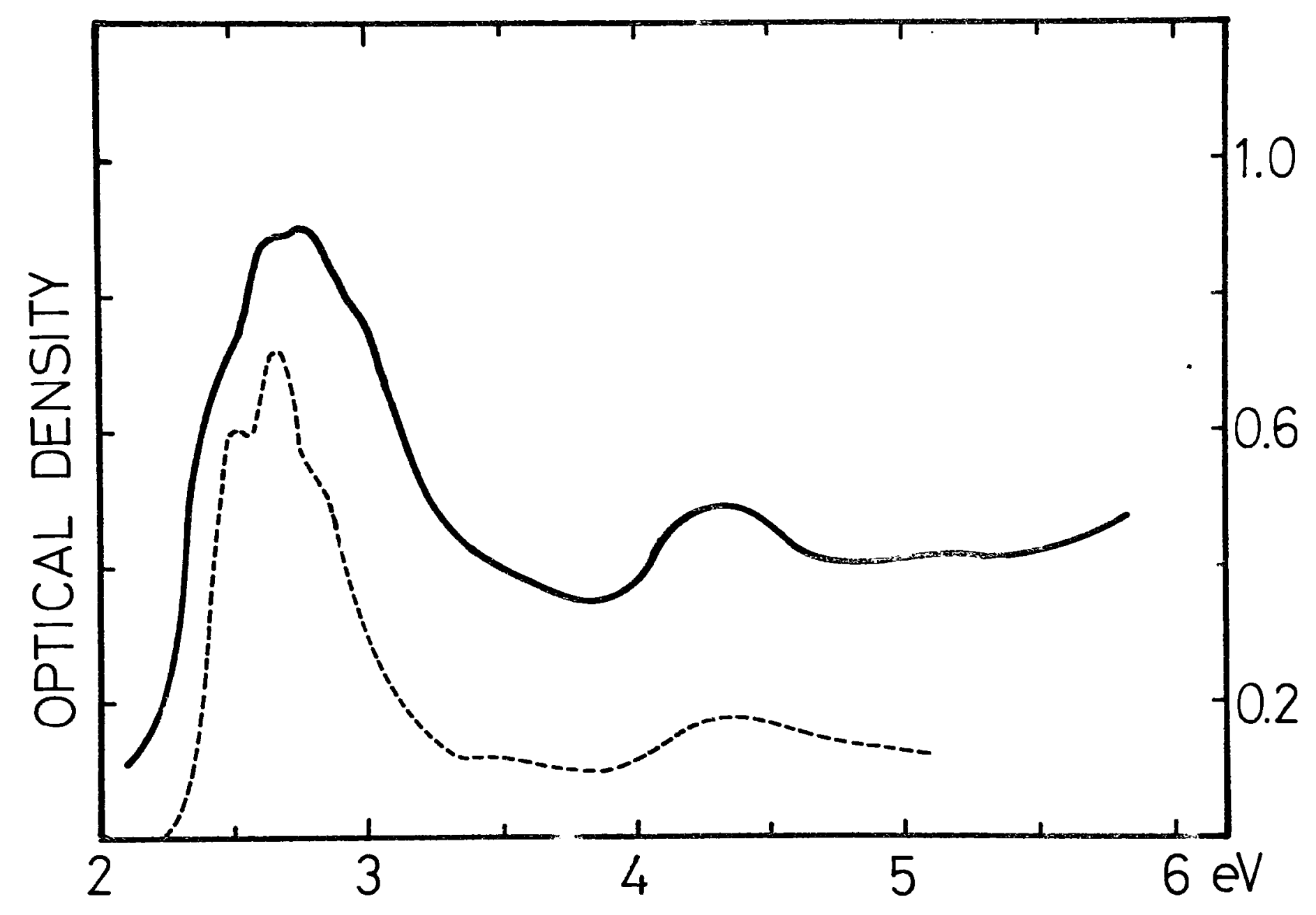


FIG. 3 b )

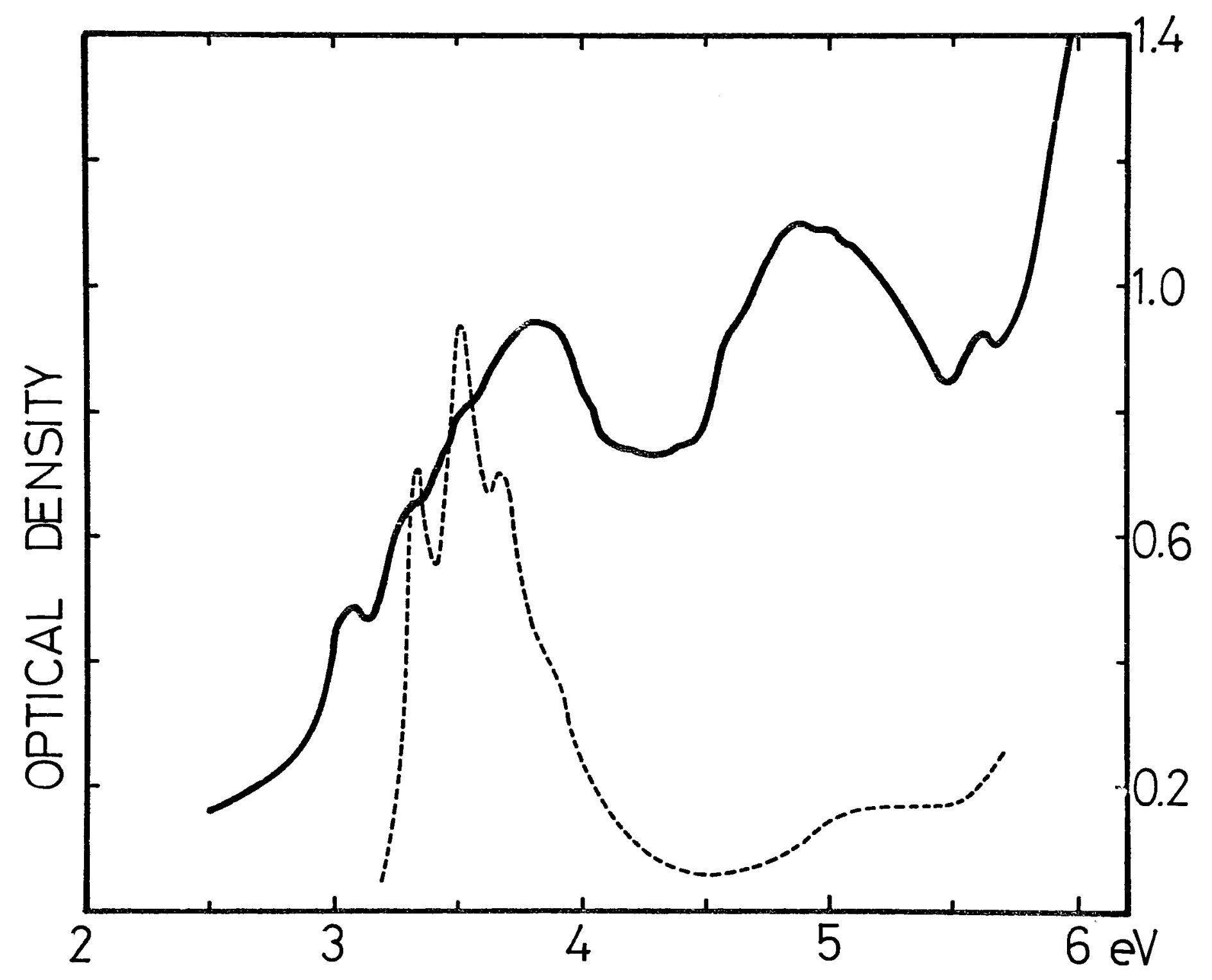

